\title{
Rational approximations for solving cauchy problems
}

\author{
Veyis Turut ${ }^{1}$ and Mustafa Bayram ${ }^{2}$ \\ ${ }^{1}$ Department of Mathematics, Faculty of Arts and Sciences, Batman University, Batman,Turkey \\ ${ }^{2}$ Department of Computer Engineering, Istanbul Gelisim University, Istanbul, Turkey
}

Received: 21 January 2016, Accepted: 8 March 2016

Published online: 16 August 2016.

\begin{abstract}
In this letter, numerical solutions of Cauchy problems are considered by multivariate Padé approximations (MPA). Multivariate Padé approximations (MPA) were applied to power series solutions of Cauchy problems that solved by using He's variational iteration method (VIM). Then, numerical results obtained by using multivariate Padé approximations were compared with the exact solutions of Cauchy problems.
\end{abstract}

Keywords: Cauchy problem, inviscid Burger's equation, multivariate padé approximaton (MPA).

\section{Introduction}

In recent times, univariate and multivariate padé approximaton have been succesfully applied to various problems in physical and engineering sciences [1-5]. "Padé approximant represents a function by the ratio of two polynomials. The coefficients of the powers occurring in the polynomials are, however, determined by the coefficients in the Taylor series expansion of the function [14]". Multivariate Padé approximation is based on univariate Padé approximation [12] but calculation methods and most of the theorems are different from each other [12]. Cuyt and her co-workers have established the uniqueness, nonuniqueness and existence results for homogeneous and nonhomogeneous multivariate Padé approximations of formal power series of several variables [15-17].

In many branches of applied sciences, the solution of a given problem is often obtained as a power series expansion. The question is then trying to approximate the function from its series expansion. A possible answer is to construct a rational function whose series expansion matches the original one as far as possible. Such rational functions are called Padé approximants [18]. In this paper, power series solutions of Cauchy problems were converted into multivariate Padé series. That is, multivariate Padé approximations were applied to the first-order partial differential equation in the form $[6]$.

$$
\begin{gathered}
u_{t}(x, t)+a(x, t) u_{x}(x, t)=\phi(x), x \in \mathfrak{R}, t>0 \\
u(x, 0)=\psi(x), x \in \mathfrak{R} .
\end{gathered}
$$

The details about the above equations can be seen in [6]. 


\section{He's variational iteration method}

The basic concepts and principles of He's variational iteration method can be seen in [7-11]. Zhou and Yao [6] obtained the following iteration formula by using the basic concepts and principles of He's variational iteration method:

$$
u_{n+1}(x, t)=u_{n}(x, t)-\int_{0}^{t}\left\{\frac{\partial u_{n}(x, \xi)}{\partial \xi}+a(x, \xi)+\frac{\partial u_{n}(x, \xi)}{\partial \xi}-\phi(x)\right\} d \xi
$$

\section{Multivariate Padé approximation}

Consider the bivariate function $f(x, y)$ with Taylor power series development

$$
f(x, y)=\sum_{i, j=0}^{\infty} c_{i j} x^{i} y^{j}
$$

around the origin [12]. The Padé approximation problem of order for $f(x, y)$ consists in finding polynomials

$$
\begin{aligned}
& p(x, y)=\sum_{k=0}^{m} A_{k}(x, y) \\
& q(x, y)=\sum_{k=0}^{n} B_{k}(x, y)
\end{aligned}
$$

such that in the power series $(f q-p)(x, y)$ the coefficients of $x^{i}$ and $y^{j}$ by solving the following equation system;

$$
\begin{aligned}
& \left\{\begin{array}{l}
C_{0}(x, y) B_{0}(x, y)=A_{0}(x, y) \\
C_{1}(x, y) B_{0}(x, y)+C_{0}(x, y) B_{1}(x, y)=A_{1}(x, y) \\
\vdots \\
C_{m}(x, y) B_{0}(x, y)+\cdots+C_{m-n}(x, y) B_{n}(x, y)=A_{m}(x, y)
\end{array}\right. \\
& \left\{\begin{array}{l}
C_{m+1}(x, y) B_{0}(x, y)+C_{m+1-n}(x, y) B_{n}(x, y)=0 \\
\vdots \\
C_{m+n}(x, y) B_{0}(x, y)+\cdots+C_{m}(x, y) B_{n}(x, y)=0
\end{array}\right.
\end{aligned}
$$

where $C_{k}=0$ if $k<0$. If the equations (8) and (9) are solved then the coefficients $A_{k}(k=0, \ldots, m)$ and $B_{k}(k=0, \ldots, n)$ are obtained. So polynomials (5) and (6) are found. polynomials $p(x, y)$ and $q(x, y)$ are called Padé equations[12]. So the multivariate Padé approximant of order $(m, n)$ for $f(x, y)$ is defined as,

$$
r_{m, n}(x, y)=\frac{p(x, y)}{q(x, y)} .
$$

Theorem 1. (Cuyt and Wuytack [12]). For every nonnegative $m$ and $n$ a unique Padé approximant of order $(m, n)$ for $f$ exists.

\section{Applications and results}

In this section multivariate Padé series solutions of Cauchy problems shall be illustrated by two examples. All the results were calculated by using the software Maple. The full VIM solutions of examples can be seen in Zhou and Yao [6]. 
Example 1.Consider the nonlinear cauchy problem [6]

$$
u_{t}(x, t)+x u_{x}(x, t)=0, x \in \mathfrak{R}, t>0
$$

$$
u(x, 0)=x^{2}, x \in \Re
$$

According to the iteration formula (3) Zhou and Yao [6] obtained following solution,

$$
u_{n}(x, t)=x^{2}\left(1-2 t+\frac{(2 t)^{2}}{2 !}-\frac{(2 t)^{3}}{3 !}+\frac{(2 t)^{4}}{4 !}-\frac{(2 t)^{5}}{5 !}+\cdots\right)
$$

The exact solution of (12) is given as $u(x, t)=x^{2} e^{-2 t}$ in [6]. If the multivariate Padé approximation is applied to equation (12) for $m=4$ and $n=2$, according to the equation system (7) and (8) the following Padé equations are obtained;

$$
p(x, t)=\frac{4 t^{4}\left(t^{2}-3 t+3\right) x^{6}}{9}
$$

and

$$
q(x, t)=\frac{4 t^{4}\left(t^{2}+3 t+3\right) x^{4}}{9} .
$$

So the multivariate Padé approximant of order $(4,2)$ for equation (12) is,

$$
r_{4,2}(x, t)=\frac{\left(t^{2}-3 t+3\right) x^{2}}{\left(t^{2}+3 t+3\right)} .
$$

If the multivariate Padé approximation is applied to equation (12) for $m=5$ and $n=2$, according to the equation system (7) and (8) the following Padé equations are obtained,

$$
p(x, t)=\frac{4 t^{6}\left(2 t^{3}-9 t^{2}+18 t-15\right) x^{6}}{135}
$$

and

$$
q(x, t)=\frac{4 t^{6}\left(t^{2}+4 t+5\right) x^{4}}{45} .
$$

So the multivariate Padé approximant of order $(5,2)$ for equation (12) is,

$$
r_{5,2}(x, t)=\frac{\left(2 t^{3}-9 t^{2}+18 t-15\right) x^{2}}{3\left(t^{2}+4 t+5\right)} .
$$

If the multivariate Padé approximation is applied to equation (12) for $m=6$ and $n=2$, according to the equation system (7) and (8) the following Padé equations are obtained,

$$
p(x, t)=0.001975308642 t^{8}\left(2 t^{4}-12 t^{3}+36 t^{2}-60 t+45\right) x^{6}
$$

and

$$
q(x, t)=0.005925925926 t^{8}\left(15+10 t+2 t^{2}\right) x^{4} .
$$


So the multivariate Padé approximant of order $(6,2)$ for equation (12) is,

$$
r_{6,2}(x, t)=0.3333333333 \frac{\left(2 t^{4}-12 t^{3}+36 t^{2}-60 t+45\right) x^{2}}{15+10 t+2 t^{2}}
$$

If the numerical results are compared, following table and figures are obtained (Table 1 and Figure 1, Figure 2, Figure 3, Figure 4. );

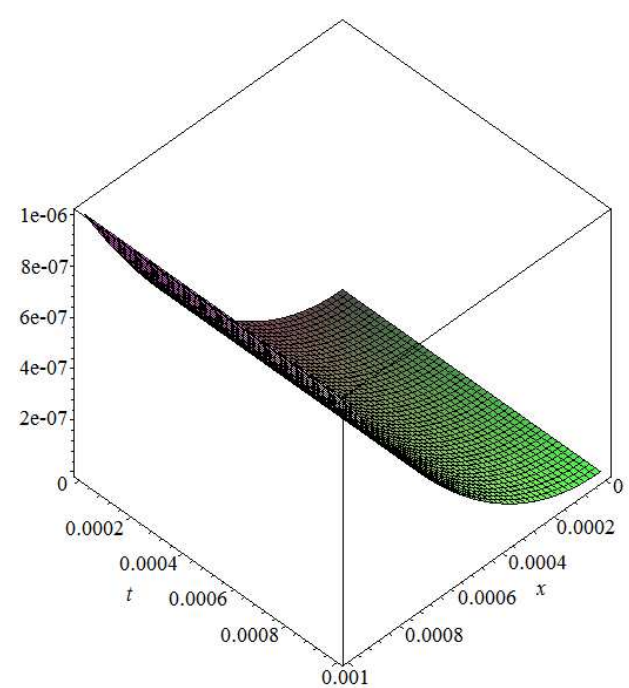

Fig. 1: Exact solution of equation (10) in Example 1.

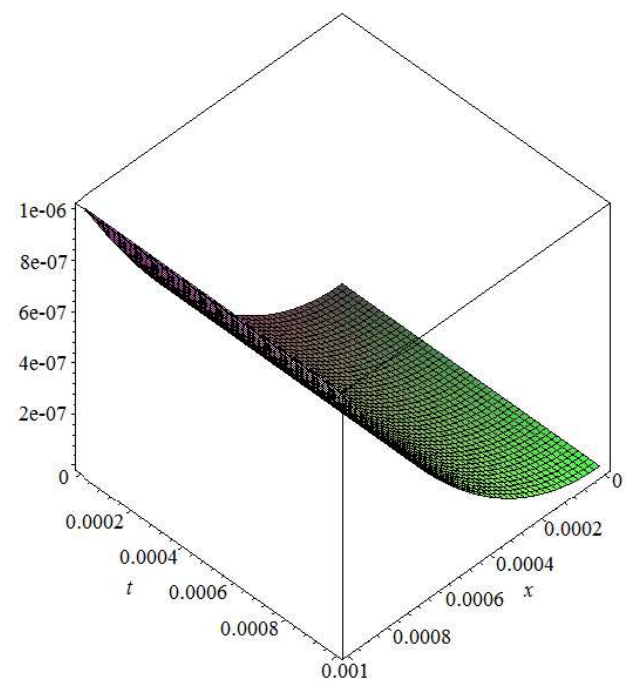

Fig. 3: $\left(r_{5,2}(x, t)\right)$, Multivariate Padé approximant of order $(5,2)$ for equation (12).

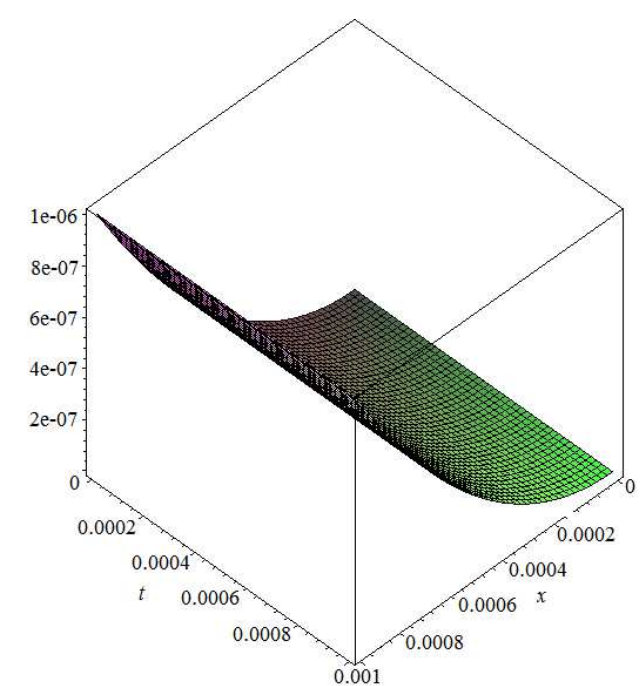

Fig. 2: $\left(r_{4,2}(x, t)\right)$, Multivariate Padé approximant of order $(4,2)$ for equation (12).

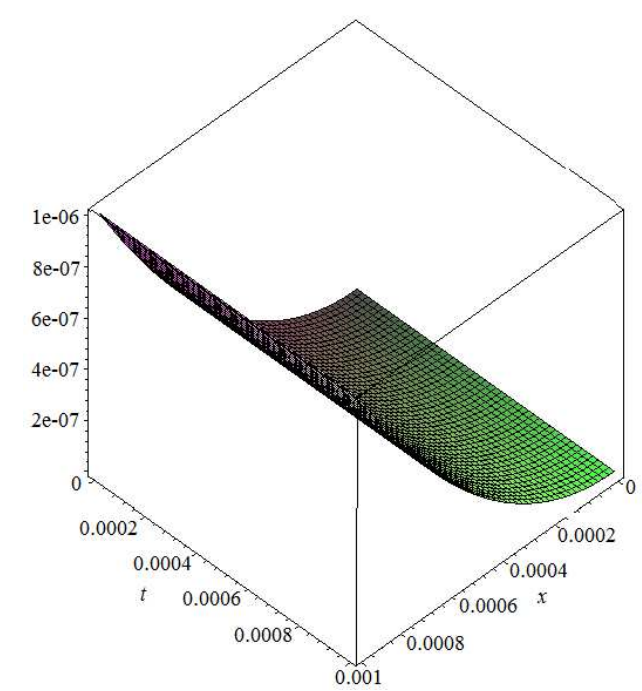

Fig. 4: $\left(r_{6,2}(x, t)\right)$, Multivariate Padé approximant of order $(6,2)$ for equation (12). 
Table 1: Comparison of Exact solution of equation (10) and MPA solutions of equation (12).

\begin{tabular}{|l|l|l|l|c|l|}
\hline$x$ & $t$ & $\begin{array}{l}\text { Exact solution } \\
u(x, t)=x^{2} e^{-2 t}\end{array}$ & $r_{4,2}(x, t)$ & $r_{5,2}(x, t)$ & $r_{6,2}(x, t)$ \\
\hline 0.001 & 0.001 & $0.9980019987 \times 10^{-6}$ & $0.9980019987 \times 10^{-6}$ & $0.9980019987 \times 10^{-6}$ & $0.9980019986 \times 10^{-6}$ \\
\hline 0.002 & 0.002 & $0.3984031957 \times 10^{-5}$ & $0.3984031957 \times 10^{-5}$ & $0.3984031957 \times 10^{-5}$ & $0.3984031956 \times 10^{-5}$ \\
\hline 0.003 & 0.003 & $0.8946161677 \times 10^{-5}$ & $0.8946161676 \times 10^{-5}$ & $0.8946161683 \times 10^{-5}$ & $0.8946161676 \times 10^{-5}$ \\
\hline 0.004 & 0.004 & 0.00001587251064 & 0.00001587251064 & 0.00001587251063 & 0.00001587251064 \\
\hline 0.005 & 0.005 & 0.00002475124584 & 0.00002475124584 & 0.00002475124585 & 0.00002475124583 \\
\hline 0.006 & 0.006 & 0.00003557058166 & 0.00003557058166 & 0.00003557058167 & 0.00003557058166 \\
\hline 0.007 & 0.007 & 0.00004831877967 & 0.00004831877967 & 0.00004831877967 & 0.00004831877966 \\
\hline 0.008 & 0.008 & 0.00006298414849 & 0.00006298414848 & 0.00006298414850 & 0.00006298414846 \\
\hline 0.009 & 0.009 & 0.00007955504362 & 0.00007955504362 & 0.00007955504363 & 0.00007955504363 \\
\hline 0.01 & 0.01 & 0.00009801986733 & 0.00009801986733 & 0.00009801986733 & 0.00009801986732 \\
\hline
\end{tabular}

Example 2.Consider the inviscid Burger's equation [6]

$$
\begin{gathered}
u_{t}(x, t)+u(x, t) u_{x}(x, t)=0, x \in \mathfrak{R}, t>0 \\
u(x, 0)=x, x \in \Re .
\end{gathered}
$$

According to the iteration formula (3) Zhou and Yao [6] obtained following solution,

$$
\begin{aligned}
& u_{4}(x, t)=x-t x+t^{2} x-t^{3} x+t^{4} x-\frac{13 t^{5} x}{15}+\frac{2 t^{6} x}{3}-\frac{t^{7} x}{29}+\frac{71 t^{8} x}{252}-\frac{86 t^{9} x}{567} \\
& +\frac{22 t^{10} x}{315}-\frac{5 t^{11} x}{189}+\frac{t^{12} x}{126}-\frac{t^{13} x}{567}+\frac{t^{14} x}{3969}-\frac{t^{15} x}{59535}
\end{aligned}
$$

The exact solution of (22) is given as $u(x, t)=\frac{x}{1+t}$ in [13]. If the multivariate Padé approximation is applied to equation (24) for $m=9$ and $n=2$, according to the equation system (7) and (8) the following Padé equations are obtained;

$$
\begin{aligned}
& p(x, t)=t^{16}\left(197313169805 t^{8}+194795648572 t^{7}+161820668856 t^{6}\right. \\
& -247699921980 t^{5}+337516192020 t^{4}-337516192020 t^{3}+337516192020 t^{2} \\
& -307283385780 t+673622025300) x^{3} / 9084507566400
\end{aligned}
$$

and

$$
q(x, t)=t^{16}\left(2482168 t^{2}+30077064 t+55305585\right) x^{2} / 745854480 .
$$

So the multivariate Padé approximant of order $(9,2)$ for equation $(24)$ is,

$$
\begin{aligned}
& r_{9,2}(x, t)=\left(197313169805 t^{8}+194795648572 t^{7}+161820668856 t^{6}\right. \\
& -247699921980 t^{5}+337516192020 t^{4}-337516192020 t^{3}+337516192020 t^{2} \\
& -307283385780 t+673622025300) x /\left(12180\left(2482168 t^{2}+30077064 t+55305585\right)\right)
\end{aligned}
$$

If the multivariate Padé approximation is applied to equation (24) for $m=11$ and $n=2$, according to the equation system (7) and (8) the following Padé equations are obtained;

$$
\begin{aligned}
& p(x, t)=t^{20}\left(3332274 t^{10}+39785435 t^{9}+280716390 t^{8}\right. \\
& +240748200 t^{7}-221629716 t^{5}+323269380 t^{4}-323269380 t^{3} \\
& \left.+323269380 t^{2}-195013980 t+762297480\right) x^{3} / 881040604500
\end{aligned}
$$


and

$$
q(x, t)=t^{20}\left(390 t^{2}+1725 t+2318\right) x^{2} / 2679075 .
$$

So the multivariate Padé approximant of order $(11,2)$ for equation (24) is,

$$
\begin{aligned}
& r_{11,2}(x, t)=\left(3332274 t^{10}+39785435 t^{9}+280716390 t^{8}\right. \\
& +240748200 t^{7}-221629716 t^{5}+323269380 t^{4} \\
& -323269380 t^{3}+323269380 t^{2}-195013980 t \\
& +762297480) x /\left(328860\left(390 t^{2}+1725 t+2318\right)\right) .
\end{aligned}
$$

If the multivariate Padé approximation is applied to equation (24) for $m=13$ and $n=2$, according to the equation system (7) and (8) the following Padé equations are obtained,

$$
\begin{aligned}
& p(x, t)=0.3378040082 .10^{-12} t^{24}\left(39150 t^{2}-261812 t^{11}\right. \\
& +0.1010766 .10^{7} t^{10}-0.1330530 .10^{7} t^{9}+0.15064245 .10^{8} t^{8} \\
& +0.9952740 .10^{7} t^{7}+0.16706088 .10^{8} t^{6}-0.23480604 .10^{8} t^{5} \\
& +0.29926260 .10^{8} t^{4}-0.29926260 .10^{8} t^{3}+0.29926260 .10^{8} t^{2} \\
& \left.-0.26637660 .10^{8} t+0.48342420 . .10^{8} t\right) x^{3}
\end{aligned}
$$

and

$$
q(x, t)=0.1110902261 t^{24}\left(147+66 t+10 t^{2}\right) x^{2} .
$$

So the multivariate Padé approximant of order $(13,2)$ for equation (24) is,

$$
\begin{aligned}
& r_{13,2}(x, t)=0.3040807638 .10^{-5}\left(39150 t^{2}-261812 t^{11}\right. \\
& +0.1010766 .10^{7} t^{10}-0.1330530 .10^{7} t^{9}+0.15064245 .10^{8} t^{8} \\
& +0.9952740 .10^{7} t^{7}+0.16706088 .10^{8} t^{6}-0.23480604 .10^{8} t^{5} \\
& +0.29926260 .10^{8} t^{4}-0.29926260 .10^{8} t^{3}+0.29926260 .10^{8} t^{2} \\
& \left.-0.26637660 .10^{8} t+0.48342420 . .10^{8} t\right) x /\left(147+66 t+10 t^{2}\right) .
\end{aligned}
$$

According to the numerical results following table and figures are obtained (Table 2 and Figure 5, Figure 6, Figure 7, Figure 8. ),

Table 2: Comparison of Exact solution of equation (22) and MPA solutions of equation (24).

\begin{tabular}{|l|l|l|l|l|l|}
\hline$x$ & $t$ & $\begin{array}{l}\text { Exact solution } \\
u(x, t)=\frac{x}{1+t}\end{array}$ & $r_{9,2}(x, t)$ & \multicolumn{1}{|c|}{$r_{11,2}(x, t)$} & $r_{13,2}(x, t)$ \\
\hline 0.001 & 0.001 & 0.0009990009990 & 0.0009990009992 & 0.0009990009989 & 0.0009990009992 \\
\hline 0.002 & 0.002 & 0.001996007984 & 0.001996007984 & 0.001996007984 & 0.001996007984 \\
\hline 0.003 & 0.003 & 0.002991026919 & 0.002991026920 & 0.002991026919 & 0.002991026918 \\
\hline 0.004 & 0.004 & 0.003984063745 & 0.003984063745 & 0.003984063744 & 0.003984063744 \\
\hline 0.005 & 0.005 & 0.004975124378 & 0.004975124379 & 0.004975124378 & 0.004975124375 \\
\hline 0.006 & 0.006 & 0.005964214712 & 0.005964214713 & 0.005964214711 & 0.005964214711 \\
\hline 0.007 & 0.007 & 0.006951340616 & 0.006951340616 & 0.006951340616 & 0.006951340616 \\
\hline 0.008 & 0.008 & 0.007936507937 & 0.007936507936 & 0.007936507936 & 0.007936507936 \\
\hline 0.009 & 0.009 & 0.008919722498 & 0.008919722496 & 0.008919722499 & 0.008919722499 \\
\hline 0.01 & 0.01 & 0.009900990099 & 0.009900990099 & 0.009900990099 & 0.009900990099 \\
\hline
\end{tabular}




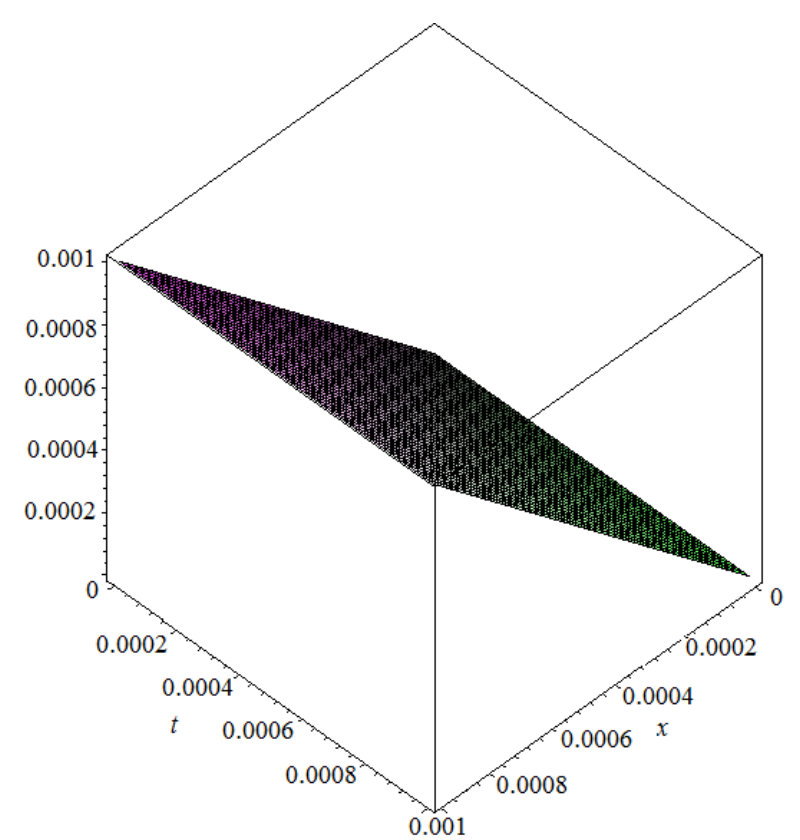

Fig. 5: Exact solution of equation (22) in Example 2.

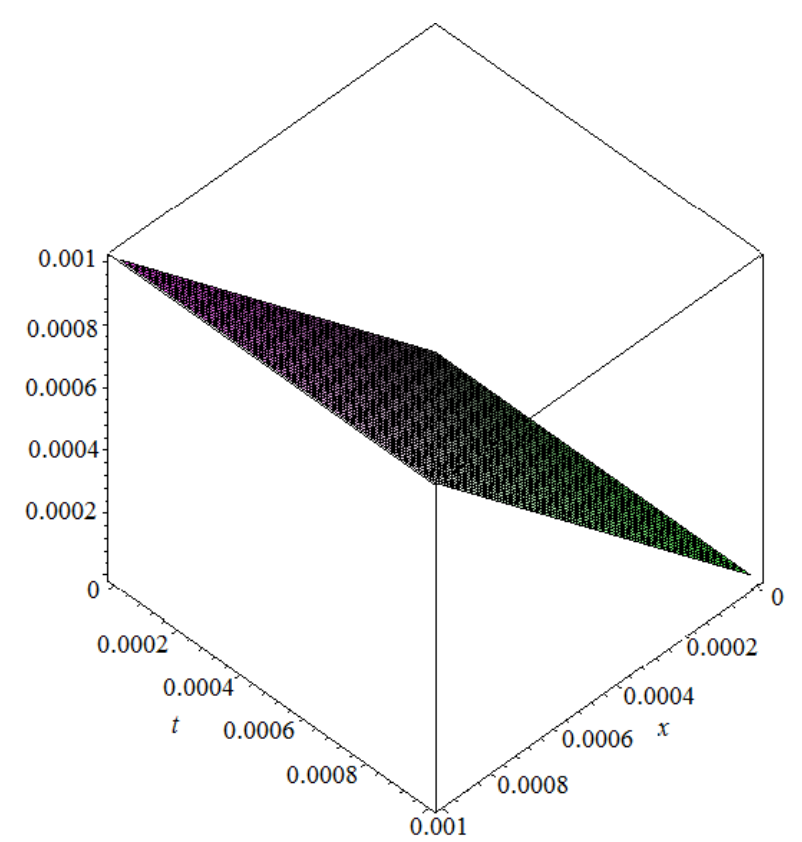

Fig. 7: $\left(r_{11,2}(x, t)\right), \quad$ Multivariate Padé approximant of order $(11,2)$ for equation (24).

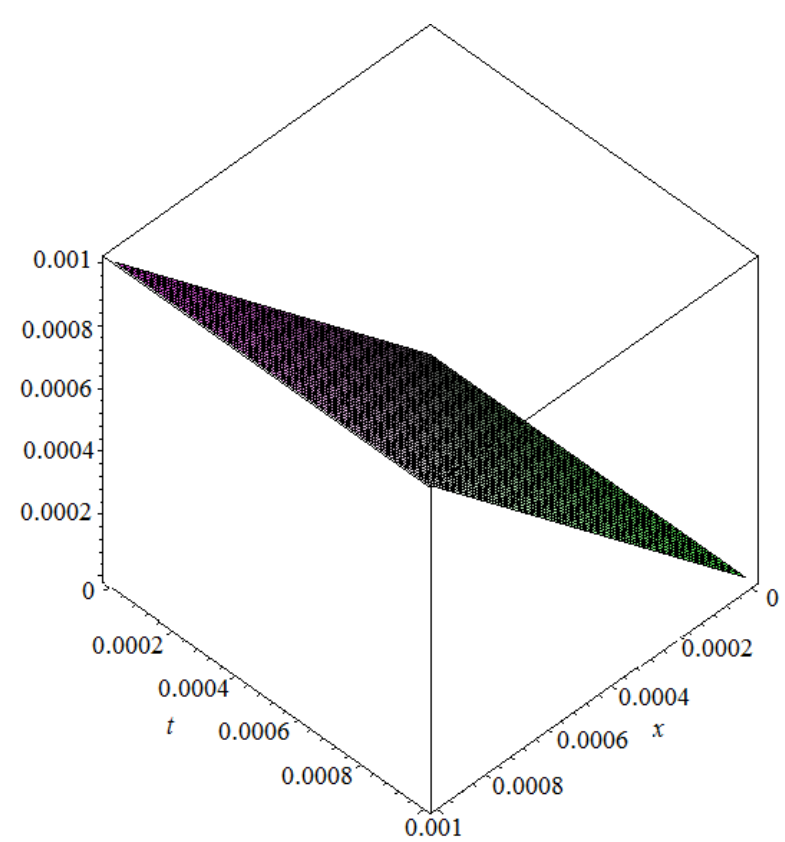

Fig. 6: $\left(r_{9,2}(x, t)\right)$, Multivariate Padé approximant of order $(9,2)$ for equation (24).

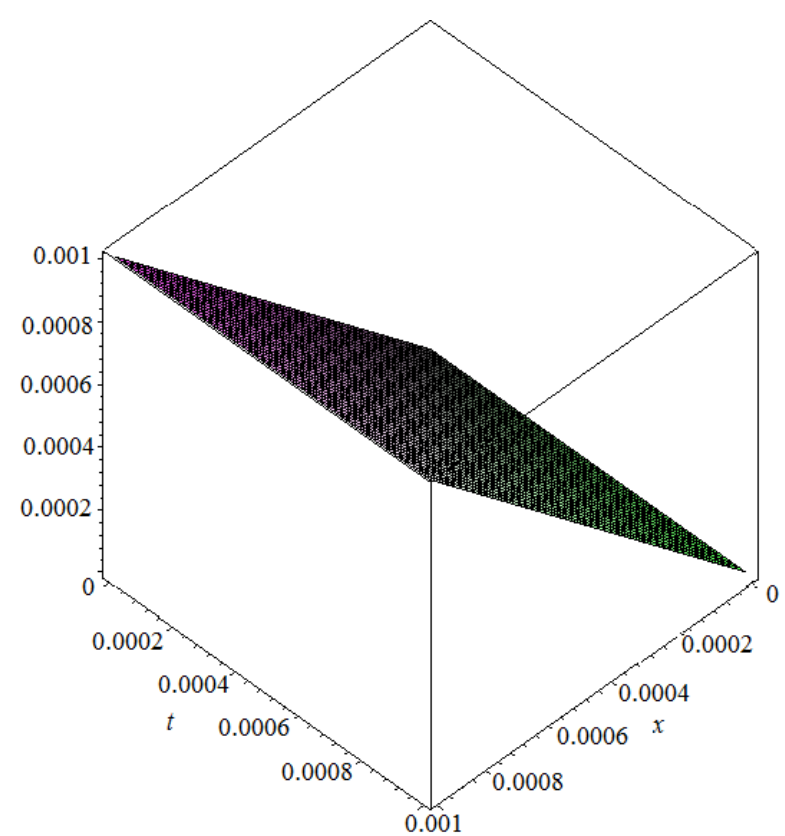

Fig. 8: $\left(r_{13,2}(x, t)\right)$, Multivariate Padé approximant of order $(13,2)$ for equation (24). 


\section{Conclusion}

In this paper, rational series solution of various kinds of Caucy problems were constructed by multivariare Padé approximation. The approximation is effective, easy to use and reliable and main benefit of the approximation is to offer rational approximation in a rapid convergent rational series form.

\section{Competing Interests}

The authors declare that they have no competing interests.

\section{Authors' Contributions}

All authors have contributed to all parts of the article. All authors read and approved the final manuscript.

\section{References}

[1] N. Guzel, M. Bayram, On the numerical solution of differential-algebraic equations with index-3, Applied Mathematics and Computation (2006), 175(2), 1320-1331.

[2] E. Celik, M. Bayram, Numerical solution of differential-algebraic equation systems and applications, Applied Mathematics and Computation (2004), 154 ( 2): 405-413.

[3] V. Turut and N Guzel., Comparing Numerical Methods for Solving TimeFractional Reaction-Diffusion Equations, ISRN Mathematical Analysis (2012), Doi:10.5402/2012/737206.

[4] V. Turut, N. Güzel, Multivariate padé approximation for solving partial differential equations of fractional order",Abstract and Applied Analysis (2013), in press.

[5] V. Turut, E. Çelik, M. Yiğider, Multivariate padé approximation for solving partial differential equations (PDE), International Journal For Numerical Methods In Fluids (2011), 66 (9):1159-1173.

[6] X. W. Zhou, L. Yao, The variational iteration method for Cauchy problems, Computers \& Mathematics with Applications (2010), 60 ( 3): 756-760.

[7] J.H. He, Approximate analytical solution for seepage flow with fractional derivatives in porous media, Comput. Methods Appl. Mech. Eng. (1998), 167: 57-68.

[8] J.H. He, Variational iteration method-a kind of non-linear analytical technique: some examples, Int. J. Non-Linear. Mech. (1999), 34: 699-708.

[9] J.H. He, Variational iteration method-some recent results and new interpretations, J. Comput. Appl. Math. (2007), 207: 3-17.

[10] J.H. He, X.H. Wu, Variational iteration method: new development and applications, Comput. Math. Appl. (2007), 54: 881-894.

[11] J.H. He, G.-C. Wu, F. Austin, The variational iteration method which should be followed, Nonlinear Sci. Lett. A (2010), 1: 1-30.

[12] A. Cuyt, L. Wuytack, Nonlinear Methods in Numerical Analysis, Elsevier Science Publishers B.V. (1987), Amsterdam

[13] Abdul-Majid Wazwaz, Partial Differential Equations and Solitary Waves Theory, Higher Education Press (2009), Beijing

[14] G. Baker , P. Graves-Morris ,Padé Approximants. Basic Theory. Encyclopedia of Mathematics and its applications: vol 13., Addison- Wpsley, Reading (1981), Massachusetts. 
[15 ] A. Cuyt, A multivariate convergence theorem of the "de Montessus de Ballore” type, J. Comput. Appl. Math. (1990), 32: $47-57$.

[16 ] A. Cuyt, K. Driver and D.S. Lubinsky, Nuttall-Pommerenke theorem for homogeneous Padé approximants, J. Comput. Appl. Math. (1996), 67: 141-146.

[17 ] A. Cuyt, K. Driver and D.S. Lubinsky, A direct approach to convergence of multivariate, non-homogeneous, Padé approximants, J. Comput. Appl. Math. (1996), 69: 353-366.

[18] C. Brezinski, Extrapolation algorithms and Padé approximations: a historical survey, Appl. Numer. Math. (1996), 20: 299-318. 